\title{
Living with COVID-19: The road ahead
}

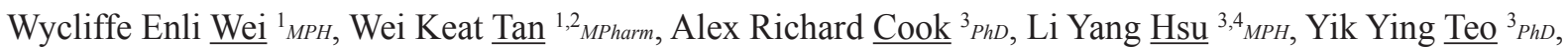
Vernon Jian Ming Lee ${ }^{1,3} P h D$

\begin{abstract}
Introduction: The COVID-19 pandemic has affected the world for more than a year, with multiple waves of infections resulting in morbidity, mortality and disruption to the economy and society. Response measures employed to control it have generally been effective but are unlikely to be sustainable over the long term.

Methods: We examined the evidence for a vaccine-driven COVID-19 exit strategy including academic papers, governmental reports and epidemiological data, and discuss the shift from the current pandemic footing to an endemic approach similar to influenza and other respiratory infectious diseases.

Results: A desired endemic state is characterised by a baseline prevalence of infections with a generally mild disease profile that can be sustainably managed by the healthcare system, together with the resumption of near normalcy in human activities. Such an endemic state is attainable for COVID-19 given the promising data around vaccine efficacy, although uncertainty remains around vaccine immunity escape in emergent variants of concern. Maintenance of non-pharmaceutical interventions remains crucial until high vaccination coverage is attained to avoid runaway outbreaks. It may also be worthwhile to de-escalate measures in phases, before standing down most measures for an endemic state. If a variant that substantially evades immunity emerges, it will need to be managed akin to a new disease threat, with pandemic preparedness and response plans.
\end{abstract}

Conclusion: An endemic state for COVID-19, characterised by sustainable disease control measures, is likely attainable through vaccination.

Ann Acad Med Singap 2021;50:619-28

Keywords: COVID-19, endemic, non-pharmaceutical interventions, transition, vaccination

\section{INTRODUCTION}

The COVID-19 pandemic has made an unprecedented impact on global morbidity, mortality and healthcare measures to contain the infection. ${ }^{1}$ Multiple waves of infections in 2020 and 2021 have resulted in significant disruptions to healthcare, economies and societies globally, with few countries able to avoid major epidemics. In the initial pandemic response, without the availability of effective therapies or vaccines, nonpharmaceutical interventions (NPIs) formed the main measures against the virus. These included border control measures, case-based and community-based NPIs - such as testing, isolating cases, contact tracing and quarantining of close contacts, mask wearing, physical distancing, and reducing of community activities. ${ }^{2}$

The availability of COVID-19 vaccines from late-2020 offers a potential tool to end the pandemic. Early data from Israel, which had vaccinated over $60 \%$ of its population, ${ }^{3}$ suggest that a vaccine-driven strategy coupled with phased reduction of NPIs could be a way to transition towards an endemic end state. ${ }^{4,5}$ Whether this level of vaccine coverage in the absence of NPIs is adequate to maintain low COVID-19 hospitalisations and deaths, especially in light of more transmissible variants, remains to be seen. In addition, there is uncertainty over the optimal approach to transition

\footnotetext{
${ }^{1}$ Communicable Diseases Division, Ministry of Health, Singapore

${ }^{2}$ Harvard T.H. Chan School of Public Health, US

${ }^{3}$ Saw Swee Hock School of Public Health, National University of Singapore, Singapore

${ }^{4}$ Yong Loo Lin School of Medicine, National University of Singapore, Singapore

Correspondence: A/Prof Vernon JM Lee, Ministry of Health, 16 College Road, College of Medicine Building, Singapore 169854.

Email: vernon_lee@moh.gov.sg
} 


\section{CLINICAL IMPACT}

\section{What is New}

- COVID-19 strategies in many countries have been focused on stringent measures. However, these are unlikely to be sustainable over the long term.

- This review examines evidence for a vaccinedriven COVID-19 exit strategy and discusses how a transition can be made from a pandemic state to an endemic one.

\section{Clinical Implications}

- More countries are ramping up vaccination efforts with the hope of transitioning from a COVID-19 pandemic state towards endemicity and restoring normalcy in society and human activities.

- One calibrated approach to transition towards endemicity would be to lift non-pharmaceutical interventions in phases while ramping up vaccination coverage, with less restrictions imposed upon vaccinated individuals.

- This will avoid unnecessary spikes in COVID-19 infection rates, deaths and strain in healthcare capacity, which can derail progression towards endemicity.

to an endemic end state. As more countries ramp up vaccination efforts, it is timely to explore the steps needed to safely transition to COVID-19 endemicity.

A vaccine-driven COVID-19 exit strategy with cautious transition is the most likely path for countries that have stringently controlled COVID-19 within their borders, such as Singapore. In this review, we examine the current evidence around a vaccine-driven COVID-19 exit strategy and how countries may shift from the current pandemic to an endemic state.

\section{ENDEMIC COVID-19}

\section{Exit strategies}

Many approaches to the control of COVID-19 have been attempted. Some countries such as Sweden had initially attempted to attain herd immunity through natural infection, although not officially stated as part of the nation's strategy, which had resulted in surges in infections, overwhelmed healthcare systems and high rates of mortality. ${ }^{6}$ No country has succeeded in achieving herd immunity for COVID-19 through natural infection alone despite continued transmission for over a year, with many pivoting away from this strategy in the face of escalating hospitalisation and mortality. ${ }^{7}$ COVID-19 infection fatality rate has been estimated at up to $15 \%$ among older adults, and fatalities have also been seen in children, highlighting how such a strategy would be extremely costly from both the health and socio-economic standpoints, particularly in vulnerable populations. ${ }^{8}$ In addition, emerging evidence on postacute COVID-19 syndrome ("long COVID") indicates possible long-term sequelae beyond 4-12 weeks in around $30 \%$ of survivors from disease onset. ${ }^{9}$ The World Health Organization (WHO) has described the attempt to reach herd immunity through natural infection without any control measures as "scientifically problematic and unethical", and cautioned against such an approach due to the "unnecessary infections, suffering and death". ${ }^{10}$

In contrast, countries like New Zealand, Australia and China have aimed for an elimination strategy through implementing extensive NPIs, including strict border closures, region-wide lockdowns, school closures and stringent physical distancing measures. However, these measures are resource-intensive and entail significant economic and societal costs that not many countries can bear, especially those without large domestic economic markets. ${ }^{11}$ Prolonged and extensive NPIs could also lead to other adverse health and social consequences, such as worsening mental and physical health problems, and exacerbating inequity in access to education and health services. ${ }^{12}$

Global eradication of COVID-19 is implausible for the foreseeable future given the high transmissibility of SARS-CoV-2 virus, the propensity for the virus to mutate and escape immunity from prior infection or vaccination, and the lack of global coordination of disease elimination measures. A pure elimination strategy is impractical in most countries reliant on flows of trade and people, such as Singapore, as the risk of importing the disease remains high while transmission continues globally. ${ }^{13}$ Rather, COVID-19 will inevitably be endemic, much like many other common respiratory diseases. ${ }^{14}$ Therefore, an exit strategy that restores normalcy, or near-normalcy, while protecting lives and minimising adverse impact on the society is much sought after. Such a strategy entails arriving at the state of substantial immunity to reduce the health burden from natural infection, which effective COVID-19 vaccines and reasonable NPIs can facilitate. It will require attaining high levels of vaccine coverage in the population and avoiding large surges in infections in the meanwhile. Ultimately it may replace the need for ongoing large-scale disease control efforts. 


\section{COVID-19 vaccine-driven endemic state}

This desired end state will entail endemicity not just in epidemiological terms, but also in societal terms with regards to the approach to COVID-19. The virus will still be circulating in the population, occasionally causing outbreaks or seasonal epidemics but with levels of disease burden that can be managed by the healthcare system without being overwhelmed. This is akin to other endemic diseases such as dengue fever and seasonal influenza which may cause severe disease, but generally do not threaten overall healthcare capacity or disrupt socio-economic activities. For countries that now place a tight lid on transmissions, such a state will allow them to shift their primary focus onto disease burden instead of a fixation on the force of infection.

Might such a state of near-normalcy be attainable for COVID-19, or would COVID-19 continue to require significant NPIs even after the vaccination roll-out is over? Current data around the COVID-19 vaccines offer insight and promise.

With a vaccine roll-out that saw over $80 \%$ of its adult population being vaccinated with the Pfizer-BioNTech BNT162b2 vaccine, in addition to some pre-existing natural immunity after earlier waves of infection, Israel saw a sharp decline in COVID-19 cases and lifted most NPIs on 1 June 2021. This included doing away with its Green Pass vaccine-certificate system, restrictions on gathering sizes and mandatory mask wearing, and also the partial opening of its borders to vaccinated travellers. ${ }^{15}$ However, trends after the lifting of NPIs showed an uptick in infected cases, primarily due to the spread of the Delta variant in unvaccinated individuals. This has led to the resumption of some NPIs such as travel advisories, reinstatement of mandatory mask wearing in schools in outbreak regions, contact tracing and quarantine. ${ }^{16}$ It is yet unknown whether the rates of severe disease and death will follow a similar upward trajectory in the longer term, or whether infections will stabilise at a low level with time.

While it is difficult to set a level of disease burden that would be acceptable to the community, comparison of COVID-19 to seasonal influenza could help to put things into perspective. Annual seasonal influenza in the US was estimated to result in up to 45 million infections, 810,000 hospitalisations and 61,000 deaths. ${ }^{17}$ The burden of disease from influenza is similar across the world, with estimated 291,243 to 645,832 global deaths annually, ${ }^{18}$ yet it is a disease we have grown to live with.

COVID-19 has been clearly a more severe disease in the absence of prior immunity (Table 1), resulting in mortality up to 40 times that of seasonal influenza in the US in 2017. Between 2010 and 2020, among adults aged above 18 years in the US, influenza vaccine coverage ranged between $37.1 \%$ and $48.4 \%$, with vaccine effectiveness between $19 \%$ and $60 \% .{ }^{19,20}$ With COVID-19 vaccination, higher coverage with more effective vaccines may substantially reduce the overall infection incidence.

Table 1. Comparison of estimated epidemiological parameters of seasonal influenza and COVID-19

\begin{tabular}{|c|c|c|c|}
\hline Parameters & Seasonal influenza & COVID-19, as of 6 May 2021 & Relative rate ratio, crude \\
\hline Basic reproduction number & $\begin{array}{c}\text { Median } 1.28(\text { IQR } 1.19-1.37)^{21} \\
\text { US: } 1.8-3.1^{22}\end{array}$ & Mean $3.32(95 \% \text { CI } 2.8-3.8)^{23}$ & 2.59 \\
\hline Incidence rate per 100,000 person days & $\begin{array}{c}\text { Global: } 1.95^{24} \\
\text { (lower respiratory tract infections, 2017) }\end{array}$ & Global: $58.5(6 \text { May } 2021)^{25}$ & 30 \\
\hline $\begin{array}{l}\text { Hospitalisation rate } \\
\text { per } 100,000 \text { person days }\end{array}$ & Global: $0.34(2017)^{24}$ & $\begin{array}{c}\text { Global: } 0.86(6 \text { May } 2021)^{25} \\
\text { US: } 1.42^{26} \\
\text { UK: } 1.36^{26} \\
\text { Israel: } 1.02^{26}\end{array}$ & 2.5 \\
\hline $\begin{array}{l}\text { Mortality rate } \\
\text { per } 100,000 \text { person days }\end{array}$ & Global: $0.005(2017)^{24}$ & Global: $0.20(6 \text { May } 2021)^{25}$ & 40 \\
\hline Vaccine coverage, at least 1 dose $(\%)$ & $\begin{array}{l}\text { US: } 37.1-48.4^{19} \\
(2010-2020)\end{array}$ & $\begin{array}{c}\text { US: } 53.3 \\
\text { UK: } 64.3 \\
\text { Israel: } 63.9 \\
(25 \text { June } 2021)^{27}\end{array}$ & - \\
\hline Vaccine effectiveness (\%) & $19-60^{20}$ & $\begin{array}{c}51-94 \\
(\text { WHO EUL })^{28}\end{array}$ & - \\
\hline
\end{tabular}

Note: Global COVID-19 incidence rates of incidence, hospitalisation and mortality were derived from cumulative rates/numbers where needed by using a global population of 7.7 billion and with a time period from 1 February 2020 to 6 May 2021.

CI: confidence interval; IQR: interquartile range; WHO EUL: World Health Organization Emergency Use Listing

Superscript numbers: Refer to numbers in REFERENCES 
Comparing COVID-19 hospitalisation and mortality rates among vaccinated persons in Israel against that of seasonal influenza in the US and England (Table 2), it is apparent that the rates of severe outcomes of COVID-19 hospitalisation and mortality are substantially reduced among vaccinated persons to a magnitude similar to that of seasonal influenza. While not a precise comparison, the magnitudes are comparable. Nevertheless, further monitoring will be required and should include examining how this data will stand up to other variants of concern, such as the Delta variant.

Earlier simulations have provided indications on the extent of vaccination coverage and vaccine effectiveness required to suppress the incidence of SARS-CoV-2 infections. One study suggested that with a basic reproduction number $\left(\mathrm{R}_{0}\right)$ of 2.5 , a vaccine coverage of $70 \%$ with vaccine effectiveness of $75 \%$ would be required to prevent an epidemic or subsequent wave of infection, and the background incidence would be stably sustained at a low level. ${ }^{32}$ The coverage required approached $100 \%$ if vaccine effectiveness fell towards $60 \%$, reinforcing the equal importance of both $\mathrm{R}_{0}$ and vaccine effectiveness. In another simulation, the projected mortality in the UK from 2022 to 2024 increased from 1,000 to 63,000 deaths when the vaccine efficacy was reduced from $85 \%$ to $60 \%$, assuming the same level of vaccine coverage of $95 \%, 90 \%$ and $85 \%$ coverage in those aged 80 years and older, 50-79 years, and 18-49 years, respectively. ${ }^{33}$ On the other hand, effective vaccines alone will be insufficient in averting a pandemic if coverage is low. ${ }^{33}$
The Pfizer-BioNTech BNT162b2 vaccine and Moderna mRNA-1273 vaccine are the most efficacious COVID-19 vaccines thus far, with efficacies of greater than $90 \%$ for symptomatic disease. ${ }^{34-36}$ The extent of indirect vaccine protection to the population has also been studied, with an increase of 20 percentage points in population coverage with Pfizer-BioNTech BNT162b2 vaccine being associated with a reduction in the positive test fraction of unvaccinated population by twofold. ${ }^{37}$ However, the Pfizer-BioNTech BNT162b2 vaccine has showed reduced effectiveness against the Delta variant, with a recent estimate at $79 \%$ for symptomatic disease. ${ }^{38}$ This does not mean that the desired endemic state cannot be achieved, as it can still reduce disease burden to a level manageable for healthcare systems and mild infections would be of less significance. In general, vaccines' effectiveness against severe disease is very high and in this respect, Johnson \& Johnson's Janssen Ad26. CoV2.S vaccine had reported effectiveness of greater than $80 \%$, Sinovac-CoronaVac vaccine $100 \%$ in its WHO Emergency Use Listing Procedure (EUL) submission, and the Pfizer-BioNTech BNT162b2 vaccine had observed effectiveness over $90 \%$ against hospitalisation with the Delta variant. ${ }^{38-40}$ As such, we can expect vaccines to markedly reduce the healthcare burden by a corresponding extent through its direct protection from hospitalisations and deaths. Nevertheless, direct protection from severe disease alone is less effective in minimising the disease burden than if the herd immunity threshold could be surpassed, perhaps together with immunity from natural infection. As disease burden is concentrated among vulnerable populations, it is particularly important to aim for high vaccination coverage among them and in persons around them.

Table 2. Comparison of disease profile of COVID-19 with and without vaccination (Israel) against that of influenza in persons aged 65 and older (US and England)

\begin{tabular}{|c|c|c|c|c|c|}
\hline & \multicolumn{5}{|c|}{ Rates among persons aged $>65$ years, in 100,000 person days } \\
\hline & \multicolumn{2}{|c|}{ COVID-193 } & \multicolumn{3}{|c|}{ Influenza, median (range) ${ }^{\mathrm{a}}$} \\
\hline & Israel (Unvaccinated) & Israel (Vaccinated) & $\begin{array}{c}\text { US }^{17} \\
(2010-2020)\end{array}$ & England ${ }^{29}$ & Singapore ${ }^{30,31}$ \\
\hline Mortality & 6.6 & 0.2 & $\begin{array}{c}0.17 \\
(0.06-0.27)\end{array}$ & $\begin{array}{c}0.18 \\
(0.08-0.53)^{b}\end{array}$ & $0.46^{\mathrm{d}}$ \\
\hline Hospitalisation & 21.7 & 0.8 & $\begin{array}{c}1.29 \\
(0.58-2.91)\end{array}$ & $0.37(0.23-0.51)^{\mathrm{c}}$ & $\begin{array}{c}0.85 \\
(0.77-1.20)^{\mathrm{e}}\end{array}$ \\
\hline \multicolumn{6}{|c|}{$\begin{array}{l}\text { a Influenza rates are of cumulative incidence rates from respective country's influenza season divided by } 365 \text { days across entire calendar year and repres } \\
\text { conservative estimates as influenza cases beyond the influenza season are few and not reported. } \\
\text { b Years 2015-2020 } \\
\text { ' Years 2018-2020 } \\
\text { ' Years 1996-2003 } \\
\text { ' Years 2010-2017 }\end{array}$} \\
\hline
\end{tabular}


However, more data on vaccine protection from the Delta variant are needed. Also, studies modelling COVID-19 disease burden have generally not considered the different protection levels for asymptomatic infection and severe disease together, and this is an important area to examine. Overall, with the current data on vaccine effectiveness and real-world evidence, a vaccine-driven end state is feasible and provides optimism that an end to the pandemic may be in sight. Maximising direct individual-level protection and achieving high populationlevel immunity from vaccination are both important to strive for, particularly as the former may be more robust against variants with higher transmissibility.

\section{Achieving the endemic end state}

With this endemic end state in mind, we considered how transition from a pandemic-response footing to an endemic state may take place. As countries continue with vaccination efforts, some level of NPIs should be maintained until an optimal level of vaccination coverage has been achieved to avoid outbreak recrudescence.

\section{How high should the target vaccination coverage be?}

The herd immunity threshold for COVID-19 was estimated to be around $65-75 \%$ assuming a basic reproduction number $\left(\mathrm{R}_{0}\right)$ of between 2.8 and 3.8, and may be as high as $90 \%$ depending on population characteristics, vaccine effectiveness and transmission dynamics. ${ }^{4,23}$ In addition, the Delta variant was observed to have a $60 \%$ increase in the effective reproduction number compared to the Beta (B.1.351) variant. ${ }^{41}$ This may imply an $\mathrm{R}_{0}$ of around 4.5-6, which would raise the required herd immunity threshold to $78-83 \%$, rendering this practically impossible to achieve with imperfect vaccine effectiveness.

While a useful reference point, achieving the herd immunity threshold in the overall population should not be the sole factor being considered. There remain reservoirs of susceptibility, including those contraindicated, resistant to vaccinations or at high-risk for spread, which could form conduits of transmission. Young children below the age of 12 , for instance, who are at the moment not recommended to receive the vaccines, will form a nucleus of susceptible individuals in which clusters could form. ${ }^{42} \mathrm{~A}$ high percentage of vaccine coverage could also mask the presence of susceptible and vulnerable fractions, which are predisposed to severe disease and outbreaks. It would be important for vaccination efforts to cover vulnerable populations such as the elderly and those with comorbid medical conditions as they would disproportionately suffer the burden of disease.

The target vaccination coverage should also consider how individual protection from severe disease would contribute to substantially reduce the COVID-19 disease burden. Vaccination targets should then be set to levels whereby the resulting infection and disease burden is manageable for a country's healthcare system in the setting of minimum NPIs. Towards this goal, a high vaccination coverage in vulnerable populations would also be crucial, beyond the overall population coverage. ${ }^{43}$ To account for these, modelling studies would be needed to inform each country's strategy, taking into consideration its own context, local epidemiology, and its healthcare capacity.

Achieving high vaccination coverage may be challenging due to several factors. First, trials have only recently commenced among children, and they have thus been left out of vaccination programmes due to the lack of safety and efficacy data in this population. This limits the maximum vaccine coverage that could be attained in the population. Vaccine hesitancy is another barrier that many jurisdictions struggle with. With COVID-19, this may be in the form of conspiracy beliefs, misinformation, fear over side effects, understating the risk of the disease and lack of confidence over the vaccine's rapid approval process. ${ }^{44}$ As of December 2019, encouraging proportions of the population strongly agreed that vaccines were important, with $60 \%$ of persons in Singapore indicated as such, with proportions in the US, UK and Israel reported at $75 \%, 59 \%$ and $46 \%$, respectively ${ }^{45}$ Nevertheless, vaccine hesitancy can affect vaccine uptake, which in turn could lead to higher rates of infections and deaths, and necessitate longer maintenance of NPIs. ${ }^{46}$ It is hence important for strong health promotion efforts to address this.

Approaches to encourage vaccine uptake can include mandating and incentivising vaccination. It is not possible to force vaccination on individuals who refuse to be vaccinated, and COVID-19 vaccines are currently provisionally authorised for pandemic use. ${ }^{47} \mathrm{~A}$ more targeted and educational approach could be to incorporate COVID-19 vaccination into the standard of medical care for specific populations, to require vaccination or regular testing among employees of organisations with high risk of exposure to the infection, or to systematically provide COVID-19 vaccination through pre-existing national vaccination programmes. The latter has been effective in preventing childhood vaccine-preventable diseases. ${ }^{48}$ The use of incentives is another approach 
to increase vaccination uptake. Granting vaccinated concessions and exemptions from NPIs are justifiable on public health grounds due to the direct and indirect risk reductions conferred by the vaccination, and would need to be paired with health promotion efforts. ${ }^{49}$

\section{When can non-pharmaceutical interventions be relaxed?}

NPIs have played a key role in controlling infections. In the pandemic state, the focus has been on minimising, or even eliminating the impact of infections. This required aggressive measures. In the endemic state, most NPIs could be eased and lifted; but until high vaccination coverage is reached, there is a need to maintain them to suppress uncontrolled infection spread and minimise deaths. ${ }^{33,50}$ With low population-level immunity rates, premature lifting of NPIs - including quarantine of contacts of cases, physical distancing and mask wearing - could lead to significantly higher number of COVID-19 infections, hospitalisations and deaths. ${ }^{4}$ It would be prudent to consider the substantive removal of NPIs only after evidence-based pre-determined vaccination targets, or population-level immunity thresholds, are surpassed in the vulnerable and general populations.

\section{How shouldnon-pharmaceutical interventions berelaxed?}

While it is clear that countries need to maintain some NPIs until a significant proportion of the population has been vaccinated, what is less certain is the optimal approach and timing to relax NPIs as vaccination coverage increases (i.e. transition towards endemicity). ${ }^{51}$ A calibrated transition approach would be to maintain NPIs, with relaxed interventions for vaccinated individuals, until a pre-determined vaccination target has been achieved before gradual relaxation of most NPIs.

Relaxation of measures should be focused on vaccinated persons during the transition towards endemicity on 2 counts. Firstly, on public health grounds, vaccinated persons have substantial protection from infection and severe disease, which may be equal to or superior to the NPIs alone. Second, this provides an incentive for the uptake of vaccination, although the timing should preferably be when vaccines are accessible to all to avoid inequity in access.

The many unknowns, particularly with respect to variants of concern, warrant a period of close monitoring and calibrated relaxation even after countries achieve their vaccination targets. This ensures that the actual disease trends that unfold are as expected (through earlier modelling studies), and provides the reassurance for subsequent policy shifts. A gradual relaxation approach also avoids sudden surges in cases after lifting of NPIs, which could potentially result in high hospitalisation and death rates. Some countries such as the UK have opted for more substantial lifting of restrictions, resulting in surges in the number of cases; however, trends indicate that severe disease rates among the elderly remain suppressed by high vaccination coverage, ${ }^{38}$ and such monitoring requires further study. The optimal time point and rate for lifting NPIs will therefore need to be based on observations from various real-world settings, coupled with modelling studies and population-level preferences.

While a vaccination target informed by data and modelling would be the prerequisite for the lifting of NPIs, the progressive relaxation would also need to be informed by data confirming the success of vaccine protection. Crucially, with the relaxation of NPIs, surveillance data on the resulting infections and disease would need to be monitored to ensure that they are in accordance with the expected or modelled trends and remain manageable for the local healthcare capacity. The transition would also need to be guided by other observational evidence and qualitative assessment of a country's situation. Real-world data on the threshold for optimal vaccination coverage and effectiveness in preventing severe disease would be closely watched, such as that reported from Israel, ${ }^{3}$ but accrual of definitive evidence would unlikely be timely as countries concurrently transition. There would also need to be a shift in the narrative of how a population deals with COVID-19-from that of a dangerous pandemic to an endemic disease that everyone has to live with. A graduated transition would help the public gain confidence and acceptance as they are eased into a new approach, especially in countries that have adopted stringent measures for a protracted period.

Broadly, NPIs can be considered across 3 domains: border controls, case-based NPIs and community-based NPIs. These NPIs could then be calibrated across 3 states: the pandemic state, transition state and endemic state (Table 3).

\section{NPIs in the transition state}

In the transition state, measures should be focused on suppressing infection spread while ramping up vaccine coverage. Some border measures will still be required to minimise importing large number of new infections. Contact tracing and testing also remain important and may be enhanced to identify and isolate cases quickly, with modifications to facilitate effective and targeted operations with potentially increasing number of cases. Nevertheless, community-based NPIs could be gradually 
Table 3. Overview of measures across different states for countries adopting an elimination or close-to-elimination strategy in the pandemic state

\begin{tabular}{|c|c|c|c|}
\hline Domains & Pandemic state & Transition state & Endemic state \\
\hline General approach & $\begin{array}{l}\text { Eliminate infection with } \\
\text { aggressive and comprehensive } \\
\text { measures. }\end{array}$ & $\begin{array}{l}\text { Continued suppression of infection } \\
\text { rates and spread while achieving } \\
\text { high vaccine coverage. }\end{array}$ & $\begin{array}{l}\text { Maintain stable disease profile and } \\
\text { rates afforded by high vaccine } \\
\text { coverage. }\end{array}$ \\
\hline Testing & $\begin{array}{l}\text { Extensive coverage and } \\
\text { comprehensive testing } \\
\text { requirements. }\end{array}$ & $\begin{array}{l}\text { Continue with testing but may ease } \\
\text { for vaccinated persons. }\end{array}$ & $\begin{array}{l}\text { Not generally required except for } \\
\text { high-risk settings. Symptomatic testing } \\
\text { will likely remain for surveillance. }\end{array}$ \\
\hline Contact tracing & $\begin{array}{l}\text { Aggressive tracing and testing } \\
\text { of contacts, wide containment } \\
\text { rings. }\end{array}$ & $\begin{array}{l}\text { Focused tracing to ensure high yield } \\
\text { and effectiveness. }\end{array}$ & $\begin{array}{l}\text { Cease tracing around individual cases. } \\
\text { Outbreak investigation and management } \\
\text { reserved for high-risk settings. }\end{array}$ \\
\hline Quarantine & Dedicated quarantine facilities. & Move towards home-based quarantine. & Generally no quarantine. \\
\hline $\begin{array}{l}\text { Isolation / } \\
\text { Case management }\end{array}$ & $\begin{array}{l}\text { Facility-based isolation, } \\
\text { stringent discharge criteria. }\end{array}$ & $\begin{array}{l}\text { Move towards a period of } \\
\text { facility-based isolation followed } \\
\text { by home-based isolation. }\end{array}$ & $\begin{array}{l}\text { Self-isolation at home with medical } \\
\text { leave. }\end{array}$ \\
\hline Community measures & $\begin{array}{l}\text { Stringent restrictions and } \\
\text { requirements. }\end{array}$ & $\begin{array}{l}\text { Slow easing, particularly granted } \\
\text { to vaccinated persons. }\end{array}$ & $\begin{array}{l}\text { Minimal key measures with focus on } \\
\text { public education and messaging, and } \\
\text { personal responsibility. }\end{array}$ \\
\hline Travel & $\begin{array}{l}\text { Border controls and quarantine } \\
\text { of travellers. }\end{array}$ & $\begin{array}{l}\text { Facilitate travel initially for } \\
\text { vaccinated persons, while retaining } \\
\text { measures aimed at surveillance } \\
\text { and detection of infection. }\end{array}$ & $\begin{array}{l}\text { Minimal restrictions although less } \\
\text { intrusive requirements may still be } \\
\text { required. }\end{array}$ \\
\hline Vaccination & $\begin{array}{l}\text { Focus on priority groups, } \\
\text { achieving broad coverage. }\end{array}$ & $\begin{array}{l}\text { Maximise coverage by targeting } \\
\text { difficult-to-reach segments. }\end{array}$ & $\begin{array}{l}\text { Maintain vaccine protection, consider } \\
\text { booster vaccinations where relevant. }\end{array}$ \\
\hline
\end{tabular}

tapered down as vaccine coverage increases, especially for vaccinated individuals in low-risk settings.

\section{NPIs in the endemic state}

Herd immunity is a desirable goal before a shift to the endemic state but may not be possible as mentioned before. As long as vulnerable populations can be highly protected from severe disease with the vaccine, the resulting infections can be mitigated with a manageable healthcare burden. Countries that transition to the endemic state when a target vaccination coverage is achieved should do so cautiously, given the uncertainty of direct vaccine protection and herd immunity levels with the new variants of concern. Upon achieving endemicity, most NPIs could be lifted. Nevertheless, a differentiated approach may be required for higher-risk settings with vulnerable populations and for groups that cannot be vaccinated. This may include retaining some level of testing and key NPIs, such as mask wearing.

\section{Booster vaccines}

Vaccine-conferred immunity is pivotal for the strategy and maintenance of the endemic state. However, there is uncertainty whether immunity will prevail over time and if booster vaccinations are required. Boosters may be required if there are emergent variants that evade immunity, or if immunity wanes substantially over time. Despite reduced vaccine effectiveness, data thus far suggest that there is still substantial protection from vaccination against the current variants of concern, particularly protection from severe disease. , $39,52^{3}$ More recently, the concern has been around the Delta variant, for which a decrease in neutralising activity by vaccine-induced antibodies has been reported. ${ }^{53}$ Nevertheless, the UK has reported reassuring data that the Pfizer-BioNTech BNT162b2 vaccine continues to have vaccine effectiveness around $80 \%$ against symptomatic disease. ${ }^{38,39}$ Further monitoring and study would still be needed to confirm the findings.

On waning immunity, neutralising antibody titres have been observed to decline with time after vaccination, although there has been no indication that this declines to a level that abrogates protection. ${ }^{54}$ Other components of the immune system, such as T-cells, also contribute to long-term immunity. ${ }^{55}$ Thus far, the Pfizer-BioNTech BNT162b2 vaccine has been reported to have preserved efficacy for at least 6 months post-vaccination, ${ }^{56}$ although longer-term data would still be needed.

The critical role that vaccination protection plays in maintaining an endemic state necessitates early design 
and implementation of possible booster vaccination strategies, including what boosters to use and to acquire them early. The operational complexity for delivering booster vaccines could be comparable with the original vaccination campaign. Countries may consider preserving sufficient vaccination capacity, capability and infrastructure in case a booster vaccine would need to be rapidly deployed.

\section{Pharmaceutical interventions}

While the focus has been on vaccination due to its effectiveness in preventing infection and disease, other effective pharmaceutical interventions could also help reduce the disease burden of COVID-19. Numerous promising trials are ongoing to develop new pharmaceutical agents or repurpose existing drugs to prevent or treat COVID-19. Currently, available pharmaceutical interventions are partially effective and mostly centred around the treatment of severe disease, including agents such as dexamethasone, antivirals and monoclonal antibodies. Many are parenteral and would still require hospital admissions. ${ }^{57}$ Nevertheless, there may be oral agents in the future for treatment in the ambulatory setting that prevents severe disease and may also serve as prophylactic agents $;{ }^{58}$ this may further reduce both disease and healthcare burden. In all, effective pharmaceutical agents are important to complement NPIs and vaccination, and they will play a bigger role as more effective and accessible treatments become available.

\section{How should new variants that substantially evade vaccine-induced protection be addressed?}

Even after attaining the endemic state, the final equilibrium can still be thwarted by a new variant that can evade vaccine-induced or natural immunity substantially, and may be exacerbated if it is more virulent or transmissible. Variants need to be monitored for these parameters, and international surveillance for the emergence of such variants would be crucial. In such a scenario, endemicity may no longer hold and adopting a pandemic approach again may be required. In a way, this is akin to the approach taken for influenza, which has the usual seasonal circulation, and rare but consistent pandemic events.

\section{CONCLUSION}

There is evidence that an endemic state is attainable for COVID-19 given the promising data around vaccine efficacy, although uncertainty remains around emergent variants of concern. In the endemic state, most measures can be stepped down with societal activities at close to pre-COVID-19 levels. SARS-CoV-2 infections would be prevalent, but the health burden would be greatly moderated and manageable for the health system. Meanwhile, maintenance of NPIs remains crucial until a high level of vaccination coverage is attained, to avoid runaway outbreaks and high health and socioeconomic impact.

\section{REFERENCES}

1. Nicola M, Alsafi Z, Sohrabi C, et al. The socio-economic implications of the coronavirus pandemic (COVID-19): A review. Int J Surg 2020;78:185-93.

2. Chen JI, Yap JC, Hsu LY, et al. COVID-19 and Singapore: From early response to circuit breaker. Ann Acad Med Singap 2020;49:561-72.

3. Haas EJ, Angulo FJ, McLaughlin JM, et al. Impact and effectiveness of mRNA BNT162b2 vaccine against SARS-CoV-2 infections and COVID-19 cases, hospitalisations, and deaths following a nationwide vaccination campaign in Israel: an observational study using national surveillance data. Lancet 2021;397:1819-29.

4. Patel MD, Rosenstrom E, Ivy JS, et al. Association of simulated COVID-19 vaccination and nonpharmaceutical interventions with infections, hospitalizations, and mortality. JAMA Netw Open 2021;4:e2110782.

5. Doroshenko A. the combined effect of vaccination and nonpharmaceutical public health interventions-ending the COVID-19 pandemic. JAMA Netw Open 2021;4:e2111675.

6. Lindström M. The COVID-19 pandemic and the Swedish strategy: epidemiology and postmodernism. SSM Popul Health 2020;11:100643.

7. Claeson M, Hanson S. COVID-19 and the Swedish enigma. Lancet 2021;397:259-61.

8. Levin AT, Hanage WP, Owusu-Boaitey N, et al. Assessing the age specificity of infection fatality rates for COVID-19: systematic review, meta-analysis, and public policy implications. Eur J Epidemiol. 2020;35:1123-38.

9. Nalbandian A, Sehgal K, Gupta A, et al. Post-acute COVID-19 syndrome. Nat Med 2021;27:601-15.

10. World Health Organization. Coronavirus disease (COVID-19): Herd immunity, lockdowns and COVID-19, 31 December 2020. Availableat:https://www.who.int/news-room/q-a-detail/herd-immunitylockdowns-and-covid-19? gclid=CjwKCAjwwqaGBhBKEiwAMk-FtO YxfOMaJ61VGoS2C8A95BFXI8h0U72XmWUPS1qp5w3GUwHXzE P16hoCeoIQAvD_BwE\#x. Accessed on 25 June 2021.

11. Global Preparedness Monitoring Board. A world in disorder, 2020. Available at: https://apps.who.int/gpmb/assets/annual_report/GPMB_ AR_2020_EN.pdf. Accessed on 11 August 2021.

12. Ho RC, Tran BX, McIntyre RS. The impact of COVID-19 pandemic on global mental health: from the general public to healthcare workers. Ann Acad Med Singap 2021;50:198-9.

13. Lee A, Thornley S, Morris AJ, et al. Should countries aim for elimination in the covid-19 pandemic? BMJ 2020;370:m3410.

14. Torjesen I. Covid-19 will become endemic but with decreased potency over time, scientists believe. BMJ 2021;372:n494.

15. Ministry of Health Israel. Israel to lift all coronavirus restriction, 2021. Available at: https:/www.gov.il/en/departments/news/2305202102. Accessed on 27 June 2021. 
16. Ministry of Health, Israel. The Ministry of Health's director general signed an amendment to the health order, 23 June 2021. Available from: https://www.gov.il/en/departments/news/23062021-01. Accessed on 27 June 2021.

17. Centers for Disease Control and Prevention. Past seasons estimated influenza disease burden, 1 October 2020. Available at: https://www.cdc.gov/flu/about/burden/past-seasons.html. Accessed on 27 June 2021.

18. Iuliano AD, Roguski KM, Chang $\mathrm{HH}$, et al. Estimates of global seasonal influenza-associated respiratory mortality: a modelling study. Lancet 2018;391:1285-300.

19. Centers for Disease Control and Prevention. Flu vaccination coverage, United States, 2019-20 influenza season, 1 October 2020. Available at: https://www.cdc.gov/flu/fluvaxview/coverage1920estimates.htm. Accessed on 25 June 2021.

20. Centers for Disease Control and Prevention. CDC seasonal flu vaccine effectiveness studies, 11 December 2020. Available at: https://www.cdc.gov/flu/vaccines-work/effectiveness-studies.htm. Accessed on 25 June 2021.

21. Biggerstaff M, Cauchemez S, Reed C, et al. Estimates of the reproduction number for seasonal, pandemic, and zoonotic influenza: a systematic review of the literature. BMC Infect Dis 2014; $14: 480$.

22. Nikbakht R, Baneshi MR, Bahrampour A. Estimation of the basic reproduction number and vaccination coverage of influenza in the United States (2017-18). J Res Health Sci 2018;18:e00427.

23. Alimohamadi Y, Taghdir M, Sepandi M. Estimate of the basic reproduction number for COVID-19: A systematic review and meta-analysis. J Prev Med Public Health 2020;53:151-7.

24. GBD 2017 Influenza Collaborators. Mortality, morbidity, and hospitalisations due to influenza lower respiratory tract infections, 2017: an analysis for the Global Burden of Disease Study 2017. Lancet Respir Med 2019;7:69-89.

25. Institute for Health Metrics and Evaluation (IHME). COVID-19 mortality, infection, testing, hospital resource use, and social distancing projections, Seattle, USA; 2021. Available at: http://www.healthdata.org/covid/data-downloads. Accessed on 11 August 2021.

26. Ritchie H, Ortiz-Ospina E, Beltekian D, et al. Coronavirus (COVID-19) hospitalizations, 11 August 2021. Available at: https:/ourworldindata. org/covid-hospitalizations. Accessed on 26 June 2021.

27. Our World in Data. Coronavirus (COVID-19) vaccinations, 11 August 2021. Available at: https://ourworldindata.org/covid-vaccinations. Accessed on 11 August 2021.

28. Institute for Health Metrics and Evaluation (IHME). COVID-19 vaccine efficacy summary, 9 August 2021. Available at: http://www.healthdata.org/covid/covid-19-vaccine-efficacy-summary. Accessed on 31 July 2021.

29. Public Health England. Surveillance of influenza and other respiratory viruses in the UK: winter 2019 to 2020, June 2020. Available at: https://assets.publishing.service.gov.uk/government/ uploads/system/uploads/attachment_data/file/895233/Surveillance_ Influenza_and_other_respiratory_viruses_in_the_UK_2019_to_2020_FINAL.pdf. Accessed on 11 August 2021.

30. Chow A, Ma S, Ling A, et al. Influenza-associated deaths in tropical Singapore. Emerg Infect Dis 2006;12:114-21.

31. Ng Y, Chua LAV, Ma S, et al. Estimates of influenza-associated hospitalisations in tropical Singapore, 2010-2017: higher burden estimated in more recent years. Influenza Other Respi Viruses 2019;13:574-81.
32. Bartsch SM, O'Shea KJ, Ferguson MC, et al. Vaccine efficacy needed for a COVID-19 coronavirus vaccine to prevent or stop an epidemic as the sole intervention. Am J Prev Med 2020; 59:493-503

33. Moore S, Hill EM, Tildesley MJ, et al. Vaccination and nonpharmaceutical interventions for COVID-19: a mathematical modelling study. Lancet Infect Dis 2021;21:793-802.

34. Hall VJ, Foulkes S, Saei A, et al. COVID-19 vaccine coverage in health-care workers in England and effectiveness of BNT162b2 mRNA vaccine against infection (SIREN): a prospective, multicentre, cohort study. Lancet 2021;397:1725-35.

35. Dagan N, Barda N, Kepten E, et al. BNT162b2 mRNA Covid-19 vaccine in a nationwide mass vaccination setting. $\mathrm{N}$ Engl $\mathrm{J}$ Med 2021;384:1412-23.

36. Pilishvili T, Fleming-Dutra KE, Farrar JL, et al. Interim estimates of vaccine effectiveness of Pfizer-BioNTech and Moderna COVID-19 vaccines among health care personnel-33 U.S. Sites, January-March 2021. MMWR Morb Mortal Wkly Rep 2021; 70:753-8.

37. Milman O, Yelin I, Aharony N, et al. Community-level evidence for SARS-CoV-2 vaccine protection of unvaccinated individuals. Nat Med 2021;27:1367-9.

38. Public Health England. COVID-19 vaccine surveillance report week 25, 24 June 2021. Available at: https://assets.publishing. service.gov.uk/government/uploads/system/uploads/attachment data/file/996565/Vaccine_surveillance_report_-_week_25.pdf. Accessed on 12 August 2021.

39. Lopez Bernal J, Andrews N, Gower C, et al. Effectiveness of Covid-19 Vaccines against the B.1.617.2 (Delta) Variant. N Eng1 J Med 2021;385:585-94.

40. World Health Organization. Interim recommendations for use of the inactivated COVID-19 vaccine, CoronaVac, developed by Sinovac, 1 June 2021. Available at: https://www.who.int/publications/i/ item/WHO-2019-nCoV-vaccines-SAGE recommendation-SinovacCoronaVac-2021.1. Accessed on 13 August 2021.

41. Campbell F, Archer B, Laurenson-Schafer H, et al. Increased transmissibility and global spread of SARS-CoV-2 variants of concern as at June 2021. Euro Surveill 2021;26:2100509.

42. Moghadas SM, Fitzpatrick MC, Shoukat A, et al. Simulated identification of silent COVID-19 infections among children and estimated future infection rates with vaccination. JAMA Netw Open 2021;4:e217097.

43. Moore S, Hill EM, Dyson L, et al. Modelling optimal vaccination strategy for SARS-CoV-2 in the UK. PLoS Comput Biol 2021;17:e1008849.

44. Troiano G, Nardi A. Vaccine hesitancy in the era of COVID-19. Public Health 2021;194:245-51.

45. de Figueiredo A, Simas C, Karafillakis E, et al. Mapping global trends in vaccine confidence and investigating barriers to vaccine uptake: a large-scale retrospective temporal modelling study. Lancet 2020;396:898-908.

46. Olivera Mesa D, Hogan AB, Watson OJ, et al. Report 43: Quantifying the impact of vaccine hesitancy in prolonging the need for nonpharmaceutical interventions to control the COVID-19 pandemic, 24 March 2021. Available at: https://www.imperial.ac.uk/media/ imperial-college/medicine/mrc-gida/2021-03-24-COVID19-Report-43. pdf. Accessed on 13 August 2021.

47. World Health Organization. COVID-19 and mandatory vaccination: Ethical considerations and caveats: policy brief, 13 April 2021. Available at: https://apps.who.int/iris/handle/10665/340841. Accessed on 13 August 2021. 
48. Liew F, Wei Ang L, Cutter J, et al. Evaluation on the effectiveness of the national childhood immunisation programme in Singapore, 1982-2007. Ann Acad Med Singap 2010;39:532-41.

49. Volpp KG, Cannuscio CC. Incentives for immunity-strategies for increasing Covid-19 vaccine uptake. N Engl J Med 2021;385:e1

50. Borchering RK, Viboud C, Howerton E, et al. Modeling of future COVID-19 cases, hospitalizations, and deaths, by vaccination rates and nonpharmaceutical intervention scenarios - United States, April-September 2021. MMWR Morb Mortal Wkly Rep 2021;70:719-24.

51. European Centre for Disease Prevention and Control. Interim guidance on the benefits of full vaccination against COVID-19 for transmission and implications for non-pharmaceutical interventions, 21 April 2021. Available at: https://www.ecdc.europa. $\mathrm{eu} / \mathrm{en} /$ publications-data/interim-guidance-benefits-full-vaccinationagainst-covid-19-transmission. Accessed on 13 August 2021.

52. Abu-Raddad LJ, Chemaitelly H, Butt AA. Effectiveness of the BNT162b2 Covid-19 vaccine against the B.1.1.7 and B.1.351 variants. N Engl J Med 2021;385:187-9.

53. Liu J, Liu Y, Xia H, et al. BNT162b2-elicited neutralization of B.1.617 and other SARS-CoV-2 variants. Nature 2021;596:273-5.
54. Widge AT, Rouphael NG, Jackson LA, et al. Durability of responses after SARS-CoV-2 mRNA-1273 vaccination. N Engl J Med 2021;384:80-2.

55. Teijaro JR, Farber DL. COVID-19 vaccines: modes of immune activation and future challenges. Nat Rev Immunol 2021;21:195-7.

56. Pfizer Inc. Pfizer and BioNTech confirm high efficacy and no serious safety concerns through up to six months following second dose in updated topline analysis of landmark COVID-19 vaccine study. Available at: https://www.pfizer.com/news/press-release/press-releasedetail/pfizer-and-biontech-confirm-high-efficacy-and-no-serious. Accessed on 25 June 2021.

57. National Centre for Infectious Diseases. Treatment guidelines for COVID-19, 14 June 2021. Available at: https://www.ncid.sg/HealthProfessionals/Diseases-and-Conditions/Documents/Treatment $\% 20$ Guidelines\%20for\%20COVID-19\%20v6_FINAL\%2014\%20June.pdf. Accessed on 13 August 2021.

58. Painter GR, Natchus MG, Cohen O, et al. Developing a direct acting, orally available antiviral agent in a pandemic: the evolution of molnupiravir as a potential treatment for COVID-19. Curr Opin Virol 2021;50:17-22. Available at: https:/www.sciencedirect.com/science/ article/pii/S1879625721000663. Accessed on 13 August 2021. 